\title{
PERMUTATION POLYNOMIALS OF DEGREE 8 OVER FINITE FIELDS OF ODD CHARACTERISTIC
}

\author{
XIANG FAN
}

\begin{abstract}
This paper provides an algorithmic generalization of Dickson's method of classifying permutation polynomials (PPs) of a given degree $d$ over finite fields. Dickson's idea is to formulate from Hermite's criterion several polynomial equations satisfied by the coefficients of an arbitrary PP of degree $d$. Previous classifications of PPs of degree at most 6 were essentially deduced from manual analysis of these polynomial equations. However, these polynomials, needed for that purpose when $d>6$, are too complicated to solve. Our idea is to make them more solvable by calculating some radicals of ideals generated by them, implemented by a computer algebra system (CAS). Our algorithms running in SageMath 8.6 on a personal computer work very fast to determine all PPs of degree 8 over an arbitrary finite field of odd order $q>8$. The main result is that for an odd prime power $q>8$, a PP $f$ of degree 8 exists over the finite field of order $q$ if and only if $q \leqslant 31$ and $q \not \equiv 1(\bmod 8)$, and $f$ is explicitly listed up to linear transformations.
\end{abstract}

\section{INTRODUCTION}

Denote by $\mathbb{F}_{q}$ the finite field of order $q$, and write $\mathbb{F}_{q}^{*}=\mathbb{F}_{q} \backslash\{0\}$. Reserve the letter $x$ for the variable of the univariate polynomial ring $\mathbb{F}_{q}[x]$ over $\mathbb{F}_{q}$. An arbitrary map from $\mathbb{F}_{q}$ to itself can be represented as $\left(a \in \mathbb{F}_{q} \mapsto f(a)\right)$ by a polynomial $f$ in $\mathbb{F}_{q}[x]$. We call $f$ is a permutation polynomial $(\mathrm{PP})$ over $\mathbb{F}_{q}$ if it represents a permutation of $\mathbb{F}_{q}$.

Rooted in Hermite [12] and Dickson [4] in the nineteenth century, the study on PPs over finite fields has aroused a growing interest, partially due to its valuable applications in other areas of mathematics and engineering, such as cryptography, coding theory, combinatorial designs and so on. For example, a special class of PPs called Dickson polynomials (introduced by [4]) played a key role in Ding and Yuan's breakthrough construction [5] of a new family of skew Hadamard difference sets in combinatorics.

Although dozens of classes of PPs (with good appearance or properties) have been found (see $[17,13]$ for recent surveys), the basic problem of classification of PPs of prescribed forms is still challenging. In his pioneering thesis work [4] on PPs, L. E. Dickson discussed the classification of all PPs of a given degree $d$ over an arbitrary finite field $\mathbb{F}_{q}$. Replacing PPs by their reductions modulo $x^{q}-x$ if necessary, it is assumed that $d<q$. Up-to-date results on this classification are as follows:

- by Dickson's 1896 thesis [4] for $d \leqslant 5$ with any $q$, and for $d=6$ with any odd $q$;

- by Li, Chandler and Xiang [15] in 2010 for $d=6$ or 7 with any even $q$;

- by the author's recent [6] for $d=7$ with any odd $q$, and [8] for $d=8$ with any even $q$.

2000 Mathematics Subject Classification. 11T06, 12 Y05.

Key words and phrases. Permutation polynomial; Hermite's criterion; Carlitz conjecture; SageMath. 
The present paper contributes to this line by classifying all PPs of degree 8 over an arbitrary $\mathbb{F}_{q}$ of odd order $q>8$. More generally, we actually provide an algorithmic generalization of Dickson's method of classifying PPs of a given degree $d$ over finite fields. Dickson's idea is to formulate from Hermite's criterion several polynomial equations satisfied by the coefficients of an arbitrary PP of degree $d$. Previously known classifications of PPs of degree at most 6 were essentially deduced from manual analysis of these polynomial equations. However, these polynomials, needed for that purpose when $d>6$, are too complicated to solve. Our idea is to make them more solvable by calculating some radicals of ideals generated by them, implemented by a computer algebra system (CAS). Our algorithms running in SageMath 8.6 on a personal computer work very fast to determine all PPs of degree 8 over finite fields of odd order, as described below.

Theorem 1. For an odd prime power $q>8$, PPs of degree 8 exist over $\mathbb{F}_{q}$ if and only if

$$
q \in\{11,13,19,23,27,29,31\} .
$$

Moreover, PPs of degree 8 in normalized form over finite fields are explicitly listed in Propositions $6,7,8,9,10,11$ and 12.

It is worth to mention that all previous classifications of PPs of degree at most 7 can be recovered very quickly by similar algorithms in our approach here, with calculations implemented by a personal computer. This approach is different from that in the author's [6] classifying PPs of degree 7. Roughly speaking, [6] uses only two simple equations provided by Hermite's criterion, and its main algorithm is a brute-force search (though optimized by linear transformations), which cannot work out for degree 8 and $q>100$ in an acceptable period of time. On the contrary, the approach here will also work for degree a little larger than 8 under the support of a personal computer. We have already done some computations for degree 9 , and is writing the results in a forthcoming paper.

The structure of this paper is as follows. Section 2 establishes Algorithm 1 for explicit polynomial equations on coefficients of PPs of degree 8 by Hermite's criterion. Section 3 verifies the non-existence of PPs of degree 8 over finite fields of odd order $q>31$, by calculations of some radicals of ideals generated by polynomials provided by Algorithm 1. Section 4 explicitly lists all PPs of degree 8 in normalized form over $\mathbb{F}_{q}$ of odd order $q$ such that $8<q \leqslant 31$, by a brute-force search.

\section{HERMITE'S CRITERION}

The main tool employed by this paper (and by the above mentioned works) is Hermite's criterion for PPs over finite fields on their coefficients. Introduced by Dickson [4] as a generalization of its prime field case in Hermite [12], this criterion is usually named after Hermite, but also sometimes called Hermite-Dickson criterion. We state here an explicit version of it from [16], with the following notations specified.

Let $\mathbb{N}=\{n \in \mathbb{Z}: n \geqslant 0\}$. For $n \in \mathbb{N}$ and $f \in \mathbb{F}_{q}[x]$, denote by $\left[x^{n}: f\right]$ the coefficient of $x^{n}$ in $f(x)$. In other words, for a nonzero $f \in \mathbb{F}_{q}[x]$, we have $f(x)=\sum_{n=0}^{\operatorname{deg}(f)}\left[x^{n}: f\right] \cdot x^{n}$, where $\operatorname{deg}(f)=\max \left\{n \in \mathbb{N}:\left[x^{n}: f\right] \neq 0\right\}$. For $t \in \mathbb{R}$, let $\lfloor t\rfloor$ indicate the largest integer $\leqslant t$.

Lemma 2 (Hermite's criterion [16, Theorem 7.6]). Let $f \in \mathbb{F}_{q}[x]$. A necessary and sufficient condition for $f$ to be a PP over $\mathbb{F}_{q}$ is that

$$
\sum_{w=1}^{\left\lfloor\frac{\operatorname{deg}\left(f^{m}\right)}{q-1}\right\rfloor}\left[x^{w(q-1)}: f^{m}\right] \begin{cases}=0 & \text { for } 1 \leqslant m \leqslant q-2, \\ \neq 0 & \text { for } m=q-1 .\end{cases}
$$


Let us show how to calculate $\left[x^{n}: f^{m}\right]$ explicitly via multinomial coefficients. Consider a polynomial $f$ of degree $d$ in $\mathbb{F}_{q}[x]$. Suppose $\operatorname{gcd}(d, q)=1$ (noting that we aim for $d=8$ with an odd $q$ ). By linear transformations, we may assume that $f$ is in normalized form, i.e. $f(x)=x^{d}+\sum_{i=1}^{d-2} a_{i} x^{i}$ with all $a_{i} \in \mathbb{F}_{q}$. For integers $j, j_{1}, j_{2}, \ldots$, and $j_{d}$, define the associated multinomial coefficient as

$$
\left(\begin{array}{cl}
j \\
j_{1}, j_{2}, \ldots, j_{d}
\end{array}\right):= \begin{cases}\frac{j !}{j_{1} ! j_{2} ! \cdots j_{d} !} & \text { if } j=j_{1}+j_{2}+\cdots+j_{d} \text { and all } j_{1}, \ldots, j_{d} \geqslant 0 \\
0 & \text { otherwise. }\end{cases}
$$

By the multinomial theorem,

$$
f(x)^{m}=\sum_{\sum_{i=1}^{d-2} j_{i}+j_{d}=m}\left(\begin{array}{c}
m \\
j_{1}, j_{2}, \ldots, j_{d-2}, j_{d}
\end{array}\right)\left(\prod_{i=1}^{d-2} a_{i}^{j_{i}}\right) \cdot x^{\sum_{i=1}^{d-2} i j_{i}+d j_{d}} .
$$

Therefore,

$$
\begin{aligned}
{\left[x^{n}: f(x)^{m}\right] } & =\sum_{\substack{\sum_{i=1}^{d-2} j_{i}+j_{d}=m \\
\sum_{i=1}^{d=2} i j_{i}+d j_{d}=n .}}\left(\begin{array}{c}
m \\
j_{1}, j_{2}, \ldots, j_{d-2}, j_{d}
\end{array}\right) \prod_{i=1}^{d-2} a_{i}^{j_{i}} \\
& =\sum_{\sum_{i=1}^{d-2}(d-i) j_{i}=d m-n}\left(\begin{array}{c}
m \\
j_{1}, j_{2}, \ldots, j_{d-2}, m-\sum_{i=1}^{d-2} j_{i}
\end{array}\right) \prod_{i=1}^{d-2} a_{i}^{j_{i}} .
\end{aligned}
$$

Define a multivariate polynomial $\mathbf{H C}_{d}(q, m)$ in $\mathbb{F}_{q}\left[x_{1}, x_{2}, \ldots, x_{d-2}\right]$ (with $d-2$ variables $x_{i}$ ) as

$$
\mathbf{H C}_{d}(q, m):=\sum_{w=1}^{\left\lfloor\frac{d m}{q-1}\right\rfloor} \sum_{\sum_{i=1}^{d-2}(d-i) j_{i}=d m-w(q-1)}\left(\begin{array}{c}
m \\
j_{1}, j_{2}, \ldots, j_{d-2}, m-\sum_{i=1}^{d-2} j_{i}
\end{array}\right) \prod_{i=1}^{d-2} x_{i}^{j_{i}} .
$$

Then Hermite's criterion asserts that $f(x)=x^{d}+\sum_{i=1}^{d-2} a_{i} x^{i}$ (with all $a_{i} \in \mathbb{F}_{q}$ ) is a PP over $\mathbb{F}_{q}$ if and only if

$$
\mathbf{H C}_{d}(q, m)\left(a_{1}, a_{2}, \ldots, a_{d-2}\right) \begin{cases}=0 & \text { for } 1 \leqslant m \leqslant q-2 \\ \neq 0 & \text { for } m=q-1\end{cases}
$$

- When $q \equiv 1(\bmod d)$, no PP of degree $d$ exists over $\mathbb{F}_{q}$ because $\mathbf{H C}_{d}\left(q, \frac{q-1}{d}\right)=1$.

- If $q \neq \equiv 0,1(\bmod d)$, then $\mathbf{H C}_{d}(q, m)=0$ when $m \leqslant\left\lfloor\frac{q}{d}\right\rfloor=\left\lfloor\frac{q-1}{d}\right\rfloor<\frac{q-1}{d}$.

When $\operatorname{gcd}(d, q)=1$, previous classifications of PPs of degree $d \leqslant 6$ were essentially deduced from manual analysis of the polynomial equations $\mathbf{H C}_{d}(q, m)\left(a_{1}, a_{2}, \ldots, a_{d-2}\right)=0$ on $a_{i}$ 's provided by Hermite's criterion. Roughly speaking, $d-2$ equations $\mathbf{H C}_{d}(q, m)\left(a_{1}, a_{2}, \ldots, a_{d-2}\right)=0$ for $\left\lfloor\frac{q}{d}\right\rfloor+1 \leqslant m \leqslant\left\lfloor\frac{q}{d}\right\rfloor+d-2$ are enough to determine $\left(a_{1}, a_{2}, \ldots, a_{d-2}\right) \in \mathbb{F}_{q}^{d-2}$ when $q>d(d-2)$. However, when $d>6$, these $d-2$ polynomials $\mathbf{H C}_{d}(q, m)$ are too long to write down explicitly, let alone to solve. Our main idea is to show the non-existence of $\left(a_{1}, \ldots, a_{d-2}\right)$ for $q>d(d-2)$, by calculating some radicals of ideals generated by some $\mathbf{H C}_{d}(q, m)$, implemented by a computer algebra system (CAS) running on a personal computer. This idea works at least for $d \leqslant 8$, provided that $\operatorname{gcd}(d, q)=1$.

In practice, all algorithms of this paper runs in SageMath [18] (version 8.6), a free open-source CAS combining the power of many existing open-source packages, such as NumPy, SciPy, Sympy, Maxima, R, GAP, SingulAR and many more, into a common Python-based interface. SageMath 
uses a Python-like language, which is very readable even for those without programming experience.

For $d=8$, the multivariate polynomial $\mathbf{H C}_{8}(q, m)$ in $\mathbb{F}_{q}\left[x_{1}, x_{2}, \ldots, x_{6}\right]$ is defined as

$$
\mathbf{H C}_{8}(q, m):=\sum_{\substack{\left.7 j_{1}+6 j_{2}+5 j_{3}+4 j_{4}+3 j_{5}+2 j_{6}=8 m-n \\
n \in\left\{w(q-1): 1 \leqslant w \leqslant \frac{8 m}{q-1}\right\rfloor\right\}}}\left(\begin{array}{c}
m \\
j_{1}, j_{2}, \ldots, j_{6}, m-\sum_{i=1}^{6} \\
j_{i}
\end{array}\right) \prod_{i=1}^{6} x_{i}^{j_{i}} .
$$

Algorithm 1 realizes $\mathbf{H} \mathbf{C}_{8}(q, m)$ as a SageMath function $\operatorname{HC}(q, m)$, outputting a multivariate polynomial in $\mathbb{F}_{q}\left[x_{1}, x_{2}, \ldots, x_{6}\right]$.

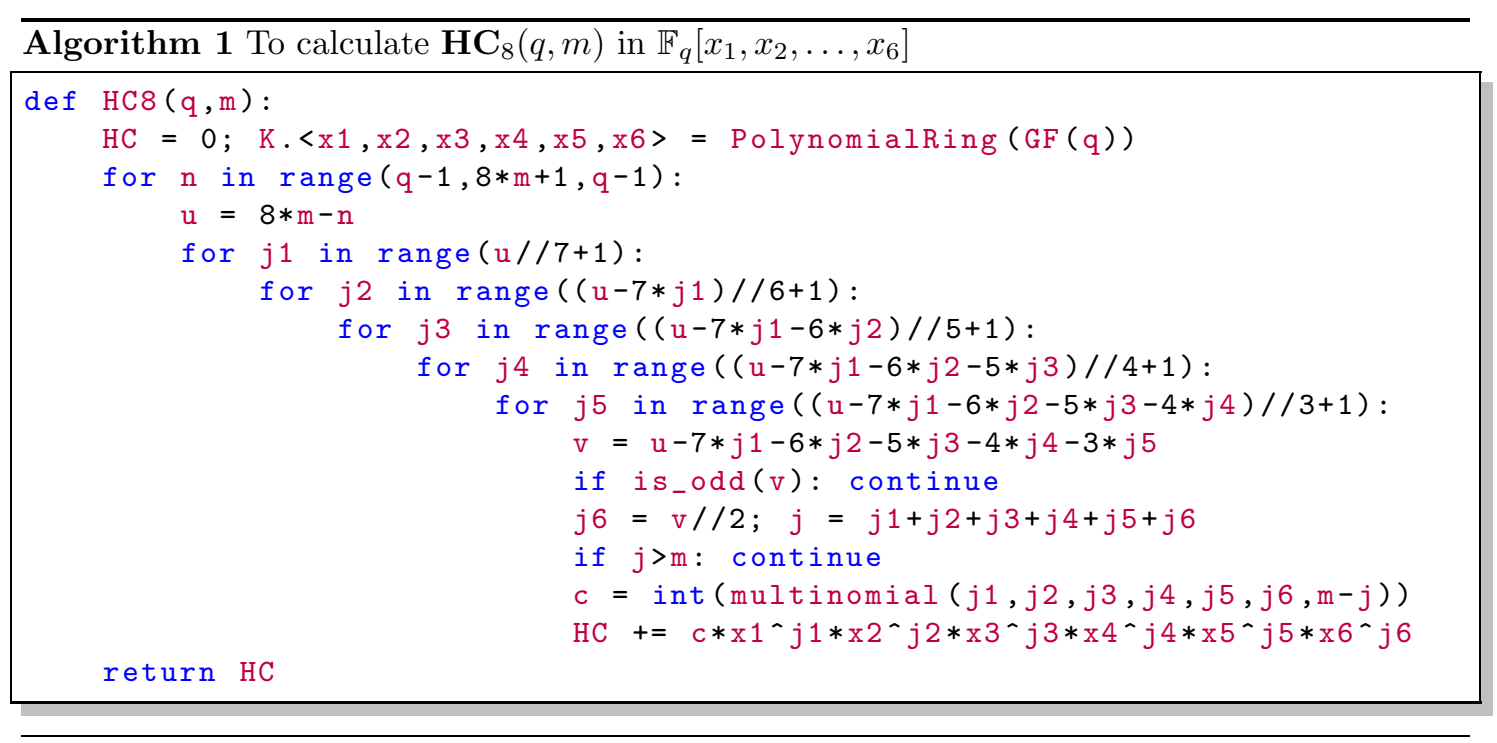

\section{NON-EXISTENCE FOR ODD $q>31$}

In an address before the Mathematical Association of America in 1966, L. Carlitz conjectured a constant $C_{n}$ for each positive even integer $n$ such that no PP of degree $n$ exists over $\mathbb{F}_{q}$ of odd order $q>C_{n}$. If $\operatorname{gcd}(n, q)=1$, it is verified by Hayes [11] with a stronger result as follows.

Lemma 3. [11, Theorem 3.4] Given a positive integer $n$, there is a constant $C_{n}$ (depending only on $n$ ) such that for any prime power $q>C_{n}$ with $\operatorname{gcd}(n, q)=1$, a PP of degree $n$ exists over $\mathbb{F}_{q}$ only if $\operatorname{gcd}(n, q-1)=1$.

Lemma 3 without the assumption $\operatorname{gcd}(n, q)=1$ is called the Carlitz-Wan conjecture, which is now a theorem by $[9,2]$. For Lemma 2 (and the Carlitz-Wan conjecture) to hold, $C_{n}$ can be taken as $n^{4}$ by von zur Gathen [10], as $n^{2}(n-2)^{2}$ by Chahal and Ghorpade [1], and as

$$
\left\lfloor\left(\frac{(n-2)(n-3)+\sqrt{(n-2)^{2}(n-3)^{2}+8 n-12}}{2}\right)^{2}\right\rfloor
$$

by the author's preprint [7]. Especially, when $n=8$, the above expression is 925 . The greatest prime power below 925 is 919 . So $C_{8}$ can be taken as 919 . 
This section will further refine the bound $C_{8}$ to 31 for the original version of Carlitz conjecture: no PP of degree 8 exists over $\mathbb{F}_{q}$ of odd order $q>31$. The main method is to calculate some radicals of ideals generated by some $\mathbf{H C}_{8}(q, m)$, with details as follows.

Consider a PP $f \in \mathbb{F}_{q}[x]$ of degree 8 over $\mathbb{F}_{q}$ of odd order $q=8 t+s$ with $1 \leqslant t \in \mathbb{Z}$ and $s \in\{3,5,7\}$. Note that $s \neq 1$ by Hermite's criterion. Without loss of loss of generality, assume that $f$ is in normalized form, i.e. $f(x)=x^{8}+\sum_{i=1}^{6} a_{i} x^{i}$ with all $a_{i} \in \mathbb{F}_{q}$. Hermite's criterion ensures that $\left(a_{1}, a_{2}, \ldots, a_{6}\right) \in \mathbb{F}_{q}^{6}$ is a vanishing point of every polynomial $\mathbf{H C}_{8}(q, m)$ such that $1 \leqslant m \leqslant q-2$, and thus of every polynomial in the radical of any ideal in $\mathbb{F}_{q}\left[x_{1}, x_{2}, \ldots, x_{6}\right]$ generated by some of them. Here the radical $\sqrt{I}$ of an ideal $I$ in a ring $R$ is defined as

$$
\sqrt{I}:=\left\{g \in R: g^{m} \in I \text { for some positive } m \in \mathbb{Z}\right\} .
$$

Especially, for $1 \leqslant k \in \mathbb{Z}$, the $\operatorname{radical} \operatorname{Rad}_{8}(q, k)$ of the ideal generated by $k$ polynomials $\mathbf{H C}_{8}(q, m)$ with $\left\lfloor\frac{q}{8}\right\rfloor+1 \leqslant m \leqslant\left\lfloor\frac{q}{8}\right\rfloor+k$ can be calculated by SageMath function $\operatorname{Rad} 8(q, k)$ in Algorithm 2.

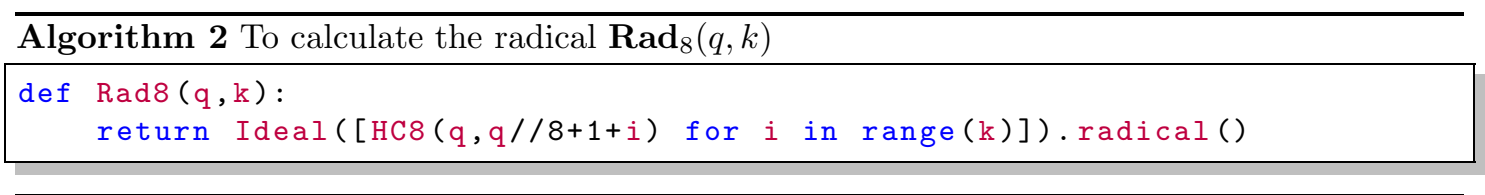

SageMath uses SingulaR [3] to implement the calculation for radicals of ideals in multivariate polynomial rings over fields, based on the algorithm of Kempers [14] in positive characteristic.

We pick out the following $\operatorname{Rad}_{8}(q, k)$ for $q>31$ given by Algorithm 2 in SageMath 8.6. Each output is of the form Ideal $\left(g_{1}, g_{2}, \ldots, g_{s}\right)$, denoting the ideal generated by $g_{1}, g_{2}, \ldots, g_{s}$ in $\mathbb{F}_{q}\left[x_{1}, x_{2}, \ldots, x_{6}\right]$. By definition, every $g_{i}$ in the output vanishes at $\left(a_{1}, a_{2}, \ldots, a_{6}\right) \in \mathbb{F}_{q}^{6}$ for any PP of the form $f(x)=x^{8}+\sum_{i=1}^{6} a_{i} x^{i}$ over $\mathbb{F}_{q}$. For each $q$, we choose a suitable $k$ to manufacture enough good $g_{i}$ 's for our purpose. Our choice of $k$ might be not as small as possible, but makes the running time of $\operatorname{Rad} 8(q, k)$ for the same result as short as possible.

- $\operatorname{Rad}_{8}(37,8)=$ Ideal(1). So no PP of degree 8 exists over $\mathbb{F}_{37}$.

- $\operatorname{Rad}_{8}(43,7)=\operatorname{Ideal}\left(x_{6}, x_{5}, x_{4}, x_{3}, x_{2}, x_{1}\right)$. Then $f(x)=x^{8}$ is clearly not a PP over $\mathbb{F}_{43}$. So no PP of degree 8 exists over $\mathbb{F}_{43}$.

- $\operatorname{Rad}_{8}(47,7)=\operatorname{Ideal}\left(x_{6}, x_{5}, x_{4}, x_{3}, x_{2}, x_{1}\right)$. So no PP of degree 8 exists over $\mathbb{F}_{47}$.

- $\operatorname{Rad}_{8}(53,7)=$ Ideal(1). So no PP of degree 8 exists over $\mathbb{F}_{53}$.

The above calculations indicate that no PP of degree 8 exists over $\mathbb{F}_{q}$ of odd order $q$ if $31<q \leqslant 53$.

- For any prime power $q \equiv 7(\bmod 8)$ with $53<q \leqslant 919$,

$$
\operatorname{Rad}_{8}(q, 7)=\operatorname{Ideal}\left(x_{6}, x_{5}, x_{3}, x_{2}, x_{1}\right) .
$$

Then $f(x)=x^{8}+a_{4} x^{4}$. Now $q=8 t+7$ with $1 \leqslant t \in \mathbb{Z}$. For $m=\left\lfloor\frac{q}{4}\right\rfloor+1=2 t+2<q-1$, by Hermite's criterion,

$$
\begin{aligned}
0 & =\mathbf{H C}_{8}(q, m)\left(0,0,0, a_{4}, 0,0\right)=\sum_{\substack{4 j_{4}=16 t+16-n \\
n \in\left\{w(8 t+6): 1 \leqslant w \leqslant\left\lfloor\frac{16 t+16}{8 t+6}\right\rfloor\right\}}}\left(\begin{array}{c}
m \\
j_{4}, m-j_{4}
\end{array}\right) a_{4}^{j_{4}} \\
& =\sum_{4 j_{4}=16 t+16-2(8 t+6)}\left(\begin{array}{c}
m \\
j_{4}, m-j_{4}
\end{array}\right) a_{4}^{j_{4}}=m a_{4} \in \mathbb{F}_{q}
\end{aligned}
$$


Note that $\operatorname{gcd}(m, q)=1$ as $q=4 m-1$, thus $a_{4}=0$. Then $f(x)=x^{8}$ is clearly not a PP over $\mathbb{F}_{q}$. So no PP of degree 8 exists over $\mathbb{F}_{q}$ if $q \equiv 7(\bmod 8)$ and $53<q \leqslant 919$.

- For any prime $q \equiv 3(\bmod 8)$ with $53<q \leqslant 919$,

$$
\operatorname{Rad}_{8}(q, 7)=\operatorname{Ideal}\left(x_{5}, x_{3}, x_{1}, x_{4} x_{6}-10 x_{2}, x_{6}^{3}-32 x_{2}\right) .
$$

- For any prime $q \equiv 5(\bmod 8)$ with $53<q \leqslant 919$,

$$
\operatorname{Rad}_{8}(q, 7)=\operatorname{Ideal}\left(x_{5}, x_{3}, x_{1}, x_{6}^{2}+\alpha(q) x_{4}, x_{4} x_{6}-10 x_{2}\right),
$$

where $\alpha(q) \in \mathbb{F}_{q}$ depends on $q$.

- For any non-prime prime power $q \equiv 3$ or $5(\bmod 8)$ with $53<q \leqslant 919$, i.e. $q=5^{3}$ or $3^{5}$,

$$
\begin{aligned}
\operatorname{Rad}_{8}\left(5^{3}, 9\right) & =\operatorname{Ideal}\left(x_{5}, x_{4}, x_{3}, x_{1}, x_{6}^{3}-2 x_{2}\right), \\
\operatorname{Rad}_{8}\left(3^{5}, 19\right) & =\operatorname{Ideal}\left(x_{5}, x_{3}, x_{1}, x_{4} x_{6}-x_{2}, x_{6}^{3}+x_{2}, x_{2} x_{6}^{2}+x_{2} x_{4}, x_{2} x_{4}^{2}+x_{2}^{2} x_{6}\right) .
\end{aligned}
$$

For any prime power $q \equiv 3$ or $5(\bmod 8)$ with $53<q \leqslant 919$, the above calculations imply that $a_{1}=a_{3}=a_{5}=0$ by Hermite's criterion. Moreover, $\mathbf{H C}_{8}(q, m)\left(0, x_{2}, 0, x_{4}, 0, x_{6}\right)$ can be calculated by the SageMath function $\operatorname{HC} 8$ new $(q, m)$ in Algorithm 3.

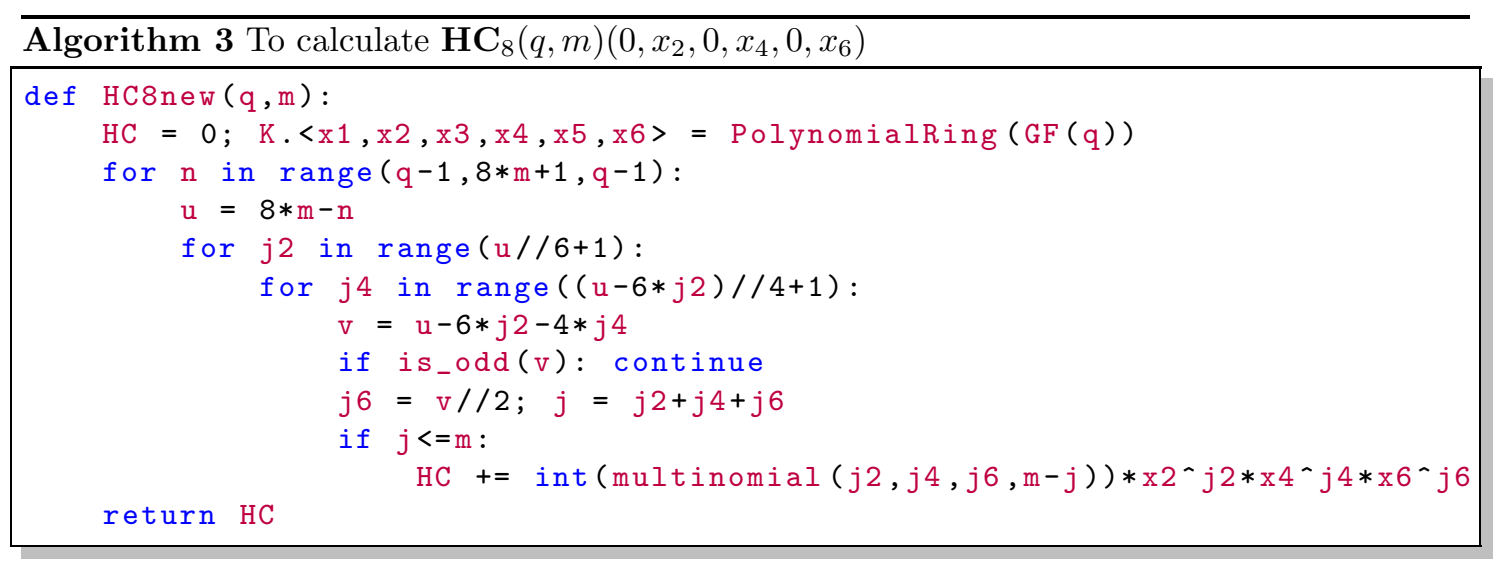

Inspired by the case of $q \equiv 7(\bmod 8)$, we try to $\operatorname{run} \operatorname{HC} 8$ new $(q, m)$ with $m=\left\lfloor\frac{q}{4}\right\rfloor+1$. After some experiments, we fortunately see that the output of the following SageMath codes in Algorithm 4, which prints $\operatorname{Ideal}\left(x_{6}, x_{5}, x_{4}, x_{3}, x_{2}, x_{1}\right)$ for every prime power $q \equiv 3$ or $5(\bmod 8)$ with $53<q \leqslant 919$, verifies the non-existence of PPs of degree 8 over $\mathbb{F}_{q}$ for these $q$.

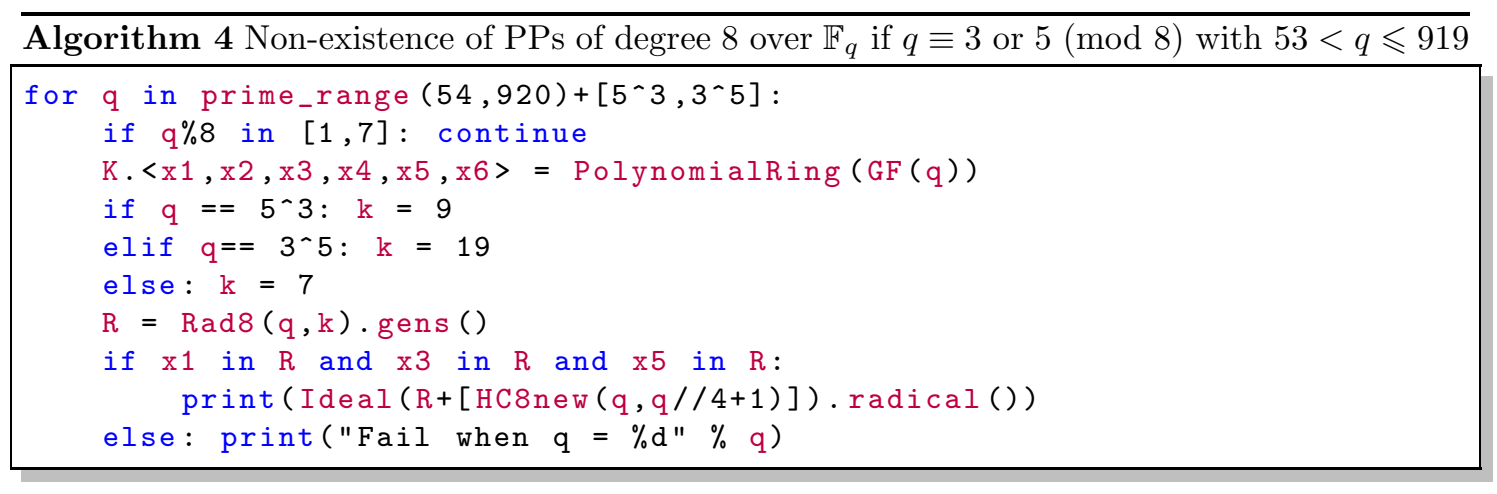


Calculations in this section for odd prime powers $q$ such that $31<q \leqslant 919$, together with Lemma 3 in which $C_{8}=919$ by [7], ensure the following Theorem 4 of non-existence.

Theorem 4. No PP of degree 8 exists over any finite field $\mathbb{F}_{q}$ of odd order $q>31$.

\section{Explicit RESUlts}

This section lists all PPs of degree 8 (in normalized form) over $\mathbb{F}_{q}$ of odd order $q>8$. Noting that $q \not \equiv 1(\bmod 8)$ by Hermite's criterion, and that $q \leqslant 31$ by Theorem 4 , we indeed have

$$
q \in\{11,13,19,23,27,29,31\} .
$$

To make the resulting list as short as possible, we first investigate the linear transformation relations among polynomials of degree 8. Two polynomials $f$ and $g$ in $\mathbb{F}_{q}[x]$ are said to be related by linear transformations (linearly related for short) if there exist $s, t \in \mathbb{F}_{q}^{*}$ and $u, v \in \mathbb{F}_{q}$ such that $g(x)=s f(t x+u)+v$. Note that linearly related $f$ and $g$ possess the same degree, and $f$ is a PP over $\mathbb{F}_{q}$ if and only if so is $g$.

Proposition 5. Let $q$ be an odd prime power. Then each polynomial of degree 8 in $\mathbb{F}_{q}[x]$ is linearly related to some $f \in \mathbb{F}_{q}[x]$ in normalized form, namely $f(x)=x^{8}+\sum_{i=1}^{6} a_{i} x^{i}$ with all $a_{i} \in \mathbb{F}_{q}$. Moreover, for another $g(x)=x^{8}+\sum_{i=1}^{6} b_{i} x^{i} \in \mathbb{F}_{q}[x]$ with all $b_{i} \in \mathbb{F}_{q}$, we have that $f$ and $g$ are linearly related if and only if $f(x)=t^{8} g\left(t^{-1} x\right)$ for some $t \in \mathbb{F}_{q}^{*}$, i.e.

$$
\left(a_{6}, a_{5}, a_{4}, a_{3}, a_{2}, a_{1}\right)=\left(t^{2} b_{6}, t^{3} b_{5}, t^{4} b_{4}, t^{5} b_{3}, t^{6} b_{2}, t^{7} b_{1}\right) .
$$

Proof. Each polynomial $h$ of degree 8 in $\mathbb{F}_{q}[x]$ can be written as $h(x)=\sum_{i=1}^{8} c_{i} x^{i}$, with all $c_{i} \in \mathbb{F}_{q}$ and $c_{8} \neq 0$. Let $f(x)=c_{8}^{-1} h\left(x-8^{-1} c_{8}^{-1} c_{7}\right)-c_{8}^{-1} h\left(-8^{-1} c_{8}^{-1} c_{7}\right)$, then $f$ is in normalized form and linearly related to $h$.

Suppose that $f(x)=x^{8}+\sum_{i=1}^{6} a_{i} x^{i}$ and $g(x)=x^{8}+\sum_{i=1}^{6} b_{i} x^{i}$ (with all $a_{i}, b_{i} \in \mathbb{F}_{q}$ ) are linearly related, namely $g(x)=s f(t x+u)+v$ with $s, t \in \mathbb{F}_{q}^{*}$ and $u, v \in \mathbb{F}_{q}$. Clearly, $s t^{8}=1$ and $8 s t^{7} u=0$, considering the coefficients of $x^{8}$ and $x^{7}$ respectively. So $s=t^{-8}$ and $u=0$. Then $g(x)=t^{-8} f(t x)+v$, where $v=g(0)-t^{-8} f(0)=0$.

Hereafter, we consider a polynomial in $\mathbb{F}_{q}[x]$ in normalized form $f(x)=x^{8}+\sum_{i=1}^{6} a_{i} x^{i}$ with all $a_{i} \in \mathbb{F}_{q}$. Algorithm 5 defines a SageMath function isPP8 $\left(q, a_{6}, a_{5}, a_{4}, a_{3}, a_{2}, a_{1}\right)$ to examine whether or not $f$ is a PP over $\mathbb{F}_{q}$. By Wan [19], it suffices to test whether or not the value set $\left\{f(c): c \in \mathbb{F}_{q}\right\}$ contains $\left\lfloor q-\frac{q-1}{8}\right\rfloor+1$ distinct values. In the following subsections, we will look for all points $\left(a_{1}, a_{2}, \ldots, a_{6}\right)$ in $\mathbb{F}_{q}^{6}$ for $f$ to be a PP over $\mathbb{F}_{q}$, up to linear transformations as indicated by Proposition 5 , for any odd prime power $q \not \equiv 1(\bmod 8)$ with $8<q \leqslant 31$, through a brute-force search optimized by a case-by-case analysis.

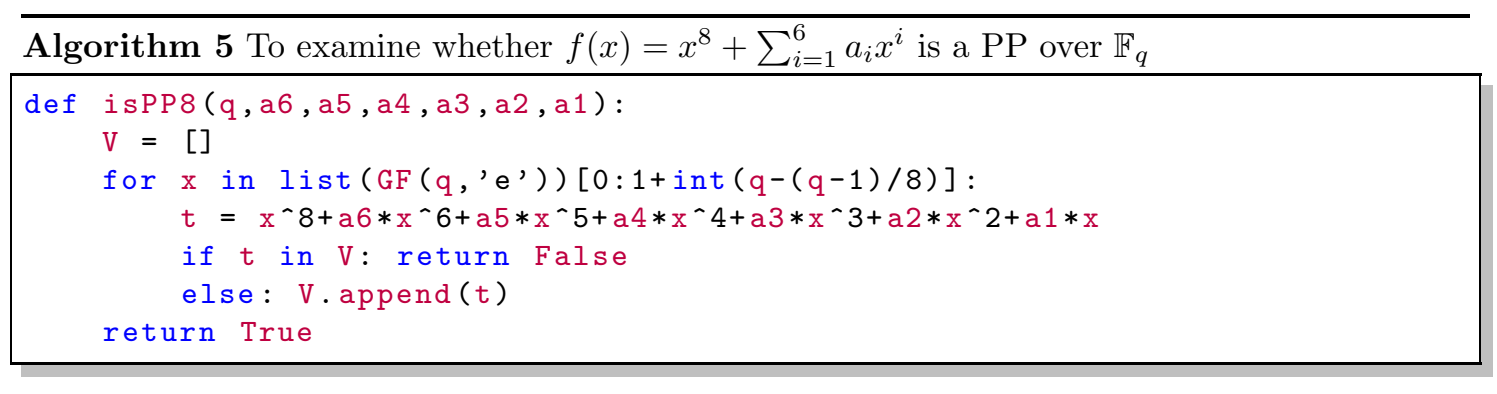


4.1. Case $q=31$. Run the following SageMath codes for polynomials in $\operatorname{Rad}_{8}(31,12)$ with at most two terms.

for $g$ in $\operatorname{Rad} 8(31,12)$.gens () :

if g.number_of_terms ( $<3$ : print $g$

The output prints $x_{6}, x_{2}^{5}-1, x_{3}^{6}-1, x_{4}^{15}-1$ and $x_{1}^{30}-1$, all of which vanish at $\left(a_{1}, a_{2}, \ldots, a_{6}\right) \in$ $\mathbb{F}_{31}^{6}$. So $a_{6}=0 \neq a_{1}$, and $a_{2}^{5}=a_{3}^{6}=a_{4}^{15}=1$.

Note that $f(x)$ is linearly related to

$$
t^{8} f\left(t^{-1} x\right)=x^{8}+t^{2} a_{6} x^{6}+t^{3} a_{5} x^{5}+t^{4} a_{4} x^{4}+t^{5} a_{3} x^{3}+t^{6} a_{2} x^{2}+t^{7} a_{1} x .
$$

As $q-1=30$ is coprime to $7, a_{1}=a^{7}$ for some $a \in \mathbb{F}_{31}^{*}$. Replacing $f(x)$ by $a^{-8} f(a x)$ if necessary, we assume $a_{1}=1$ without loss of generality. So Algorithm 6 lists PPs of degree 8 in normalized form over $\mathbb{F}_{31}$, up to linear transformations.

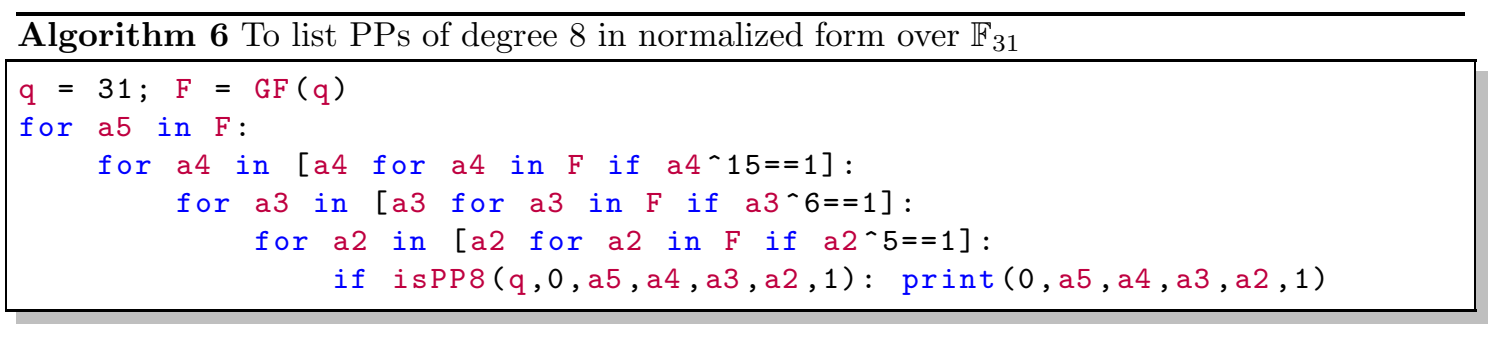

The output of Algorithm 6 is $(0,19,25,6,2,1)$, which gives the following Proposition 6 .

Proposition 6. All PPs of degree 8 in normalized form over $\mathbb{F}_{31}$ are exactly

$$
x^{8}+19 t^{3} x^{5}+25 t^{4} x^{4}+6 t^{5} x^{3}+2 t^{6} x^{2}+t^{7} x,
$$

with $t$ running through $\mathbb{F}_{31}^{*}$.

4.2. Case $q=29$. Run the following SageMath codes for polynomials in $\operatorname{Rad}_{8}(29,7)$ with at most three terms.

for $g$ in $\operatorname{Rad} 8(29,7)$.gens () :

if $g . n_{u m b e r}$ of_terms ()$<4$ : print $g$

The output prints $x_{6}^{2}-9 x_{4}, x_{1}^{8}+4 x_{1}^{4}-5, x_{2}^{15}-x_{2}$ and $x_{3}^{29}-x_{3}$, all of which vanish at $\left(a_{1}, a_{2}, \ldots, a_{6}\right) \in \mathbb{F}_{29}$. So $a_{4}=a_{6}^{2} / 9, a_{1}^{8}+4 a_{1}^{4}=5$ and $a_{2}^{15}=a_{2}$.

As $q-1=28$ is coprime to 3 , without loss of generality we can assume $a_{5} \in\{0,1\}$ (by linear transformations if necessary). So Algorithm 7 lists PPs of degree 8 in normalized form over $\mathbb{F}_{29}$, up to linear transformations.

The output of Algorithm 7 prints:

$$
(0,0,0,0,0,4),(0,0,0,0,0,10),(0,0,0,0,0,19),(0,0,0,0,0,25),(26,1,1,4,20,1) .
$$

Note that $\left\{4 t^{7}: t \in \mathbb{F}_{29}^{*}\right\}=\{4,10,19,25\}$. So we get the following Proposition 7 .

Proposition 7. All PPs of degree 8 in normalized form over $\mathbb{F}_{29}$ are exactly

$$
x^{8}+4 t^{7} x, \quad x^{8}+26 t^{2} x^{6}+t^{3} x^{5}+t^{4} x^{4}+4 t^{5} x^{3}+20 t^{6} x^{2}+t^{7} x,
$$

with $t$ running through $\mathbb{F}_{29}^{*}$. 


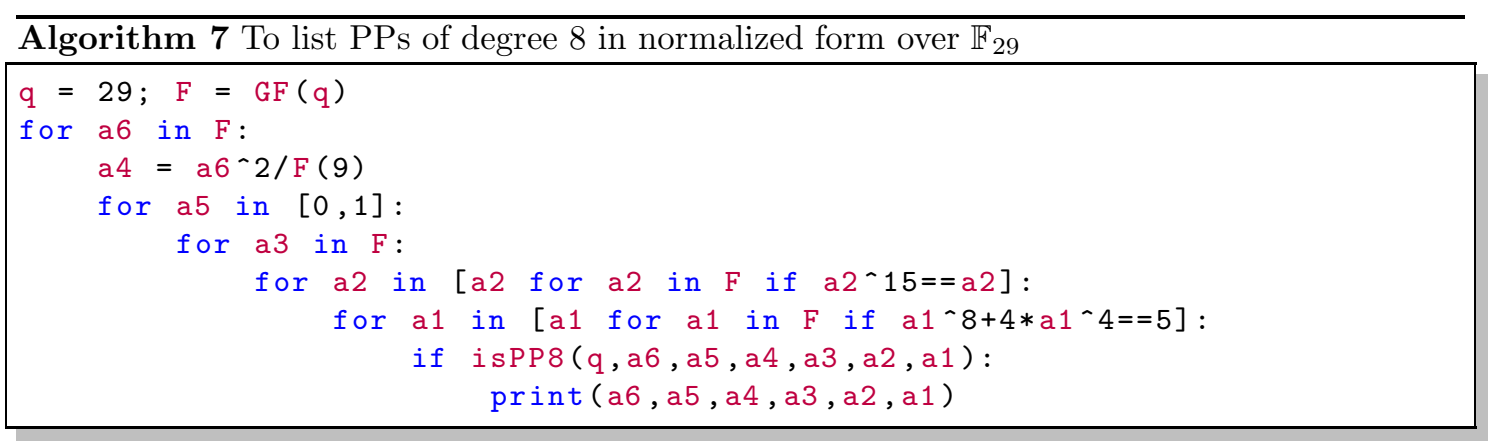

4.3. Case $q=27$. Note that $\left(a_{1}, a_{3}\right) \neq(0,0)$ by the output of the following SageMath codes.

K. $\langle\mathrm{x} 1, \mathrm{x} 2, \mathrm{x} 3, \mathrm{x} 4, \mathrm{x} 5, \mathrm{x} 6\rangle=$ PolynomialRing $(\mathrm{GF}(27))$

Ideal ([HC8 $(27,4+i)$ for $i$ in range(10)]+[x1,x3]).radical()

The output is Ideal(1), which indicates that polynomials $x_{1}$ and $x_{3}$ cannot both vanish at $\left(a_{1}, a_{2}, \ldots, a_{6}\right) \in \mathbb{F}_{27}$. So we can make the following assumptions on $\left(a_{1}, a_{2}, \ldots, a_{6}\right)$, by linear transformations if necessary.

- Assume $a_{1} \in\{0,1\}$ as $q-1=26$ is coprime to 7 .

- When $a_{1}=0$ (and thus $a_{3} \neq 0$ ), assume $a_{3}=1$, as $q-1=26$ is coprime to 5 .

Note that $a_{2}=-a_{6}^{3}$ since $\mathbf{H C}_{8}(27,4)=x_{6}^{3}+x_{2}$. So Algorithm 8 lists PPs of degree 8 in normalized form over $\mathbb{F}_{27}$ up to linear transformations, and Proposition 8 is read off from its output.

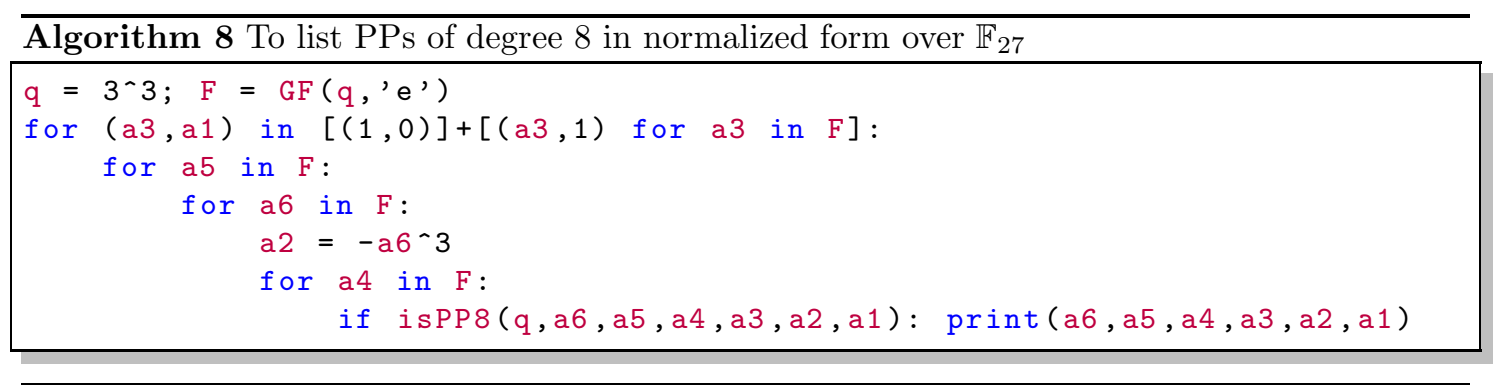

Proposition 8. All PPs of degree 8 in normalized form over $\mathbb{F}_{27}$ are exactly those of the form $x^{8}+\sum_{i=1}^{6} t^{8-i} a_{i} x^{i}$, with $t \in \mathbb{F}_{27}^{*}$ and $\left(a_{6}, a_{5}, a_{4}, a_{3}, a_{2}, a_{1}\right)$ listed as follows:

$$
\begin{array}{lll}
(1,0,2,1,2,0), & (1,1,2,1,2,0), \\
\left(e^{2}, 2 e, 2 e^{3}, e^{10}, 2 e^{6}, 1\right), & \left(e^{6}, 2 e^{3}, 2 e^{9}, e^{4}, e^{5}, 1\right), & \left(2 e^{5}, 2 e^{9}, 2 e, e^{12}, 2 e^{2}, 1\right), \\
\left(e^{2}, 2 e^{4}, e^{7}, e^{6}, 2 e^{6}, 1\right), & \left(e^{6}, 2 e^{12}, 2 e^{8}, 2 e^{5}, e^{5}, 1\right), & \left(2 e^{5}, 2 e^{10}, e^{11}, e^{2}, 2 e^{2}, 1\right), \\
\left(e^{4}, 2 e^{2}, e^{7}, e^{4}, 2 e^{12}, 1\right), & \left(e^{12}, 2 e^{6}, 2 e^{8}, e^{12}, 2 e^{10}, 1\right), & \left(e^{10}, e^{5}, e^{11}, e^{10}, 2 e^{4}, 1\right), \\
\left(2 e^{4}, e^{10}, 2 e^{12}, 2 e^{11}, e^{12}, 1\right), & \left(2 e^{12}, e^{4}, 2 e^{10}, 2 e^{7}, e^{10}, 1\right), & \left(2 e^{10}, e^{12}, 2 e^{4}, e^{8}, e^{4}, 1\right) .
\end{array}
$$


4.4. Case $q=23$. Run the following SageMath codes for polynomials in $\operatorname{Rad}_{8}(23,9)$ with at most four terms.

for $g$ in $\operatorname{Rad}(23,9)$. gens () :

if $g$. number_of_terms ()$<5$ : print $g$

The output prints $x_{6}, x_{4} x_{5}^{2}-11 x_{3}^{2}+x_{2} x_{4}+x_{1} x_{5}, x_{5}^{22}-1, x_{4}^{22}-1, x_{3}^{22}-1$ and $x_{1}^{22}-1$, all of which vanish at $\left(a_{1}, a_{2}, \ldots, a_{6}\right) \in \mathbb{F}_{23}$. So $a_{6}=0 \neq a_{j}$ for $j \in\{1,3,4,5\}$, and $a_{4} a_{5}^{2}-11 a_{3}^{2}+a_{2} a_{4}+a_{1} a_{5}=$ 0 .

As $q-1=22$ is coprime to 3 , without loss of generality we can assume $a_{5}=1$ (by linear transformations if necessary). So Algorithm 9 lists PPs of degree 8 in normalized form over $\mathbb{F}_{23}$ up to linear transformations, and Proposition 9 is read off from its output.

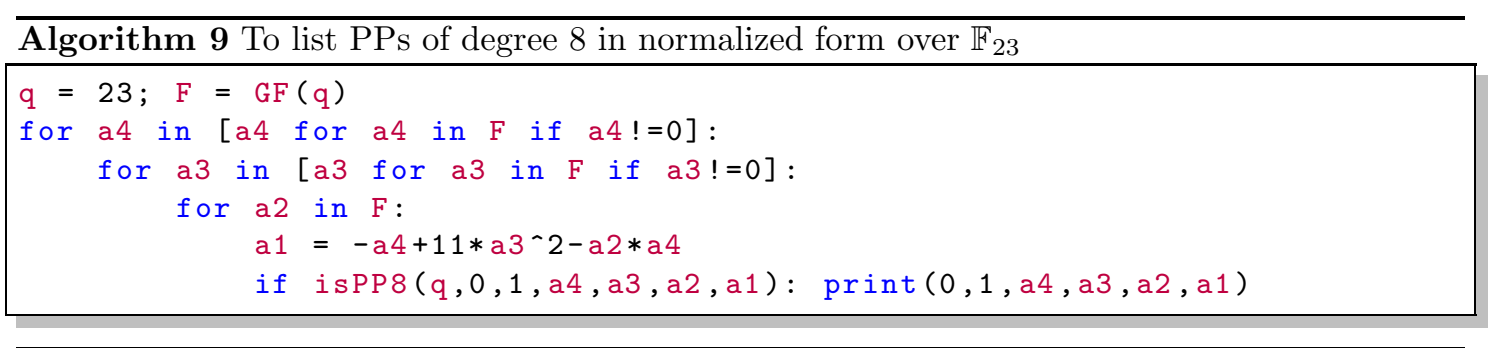

Proposition 9. All PPs of degree 8 in normalized form over $\mathbb{F}_{23}$ are exactly those of the form $x^{8}+t^{3} x^{5}+t^{4} a_{4} x^{4}+t^{5} a_{3} x^{3}+t^{6} a_{2} x^{2}+t^{7} a_{1} x$, with $t \in \mathbb{F}_{23}^{*}$ and $\left(a_{4}, a_{3}, a_{2}, a_{1}\right)$ listed as follows:

$$
\begin{array}{llll}
(11,1,12,6), & (11,1,21,22), & (15,8,16,12), & (15,16,13,7), \\
(16,8,7,1), & (19,5,10,20), & (20,12,2,6) . &
\end{array}
$$

4.5. Case $q=19$. Note that $\left(a_{1}, a_{3}\right) \neq(0,0)$ by the output of the following SageMath codes.

K. $\langle\mathrm{x} 1, \mathrm{x} 2, \mathrm{x} 3, \mathrm{x} 4, \mathrm{x} 5, \mathrm{x} 6\rangle=$ PolynomialRing (GF (19))

Ideal $([\mathrm{HC} 8(19,3+i)$ for $i$ in range $(7)]+[x 3, x 1])$.radical ()

The output is Ideal(1), which indicates that polynomials $x_{1}$ and $x_{3}$ cannot both vanish at $\left(a_{1}, a_{2}, \ldots, a_{6}\right) \in \mathbb{F}_{19}$. So we can make the following assumptions on $\left(a_{1}, a_{2}, \ldots, a_{6}\right)$, by linear transformations if necessary.

- Assume $a_{1} \in\{0,1\}$ as $q-1=18$ is coprime to 7 .

- When $a_{1}=0$ (and thus $a_{3} \neq 0$ ), assume $a_{3}=1$, as $q-1=18$ is coprime to 5 .

Note that $a_{2}=-a_{6}^{3} / 3-a_{5}^{2}-2 a_{4} a_{6}$ since

$$
\mathbf{H C}_{8}(19,3)=x_{6}^{3}+3 x_{5}^{2}+6 x_{4} x_{6}+3 x_{2} .
$$

So Algorithm 10 lists PPs of degree 8 in normalized form over $\mathbb{F}_{19}$ up to linear transformations. There are exactly 48 linearly related classes of PPs of degree 8 over $\mathbb{F}_{19}$ as listed in Proposition 10, corresponding to 48 outputting tuples of Algorithm 10.

Proposition 10. All PPs of degree 8 in normalized form over $\mathbb{F}_{19}$ are exactly those of the form $x^{8}+\sum_{i=1}^{6} t^{8-i} a_{i} x^{i}$, with $t \in \mathbb{F}_{19}^{*}$ and $\left(a_{6}, a_{5}, a_{4}, a_{3}, a_{2}, a_{1}\right)$ listed as follows:
$(0,1,3,1,18,0)$,
$(8,1,7,1,14,0)$,
$(15,1,1,1,3,0)$,
$(9,9,18,0,17,1)$
$(17,16,2,0,8,1)$,
$(4,7,17,1,9,1)$
$(8,16,10,1,15,1)$,
$(15,4,9,1,14,1)$ 


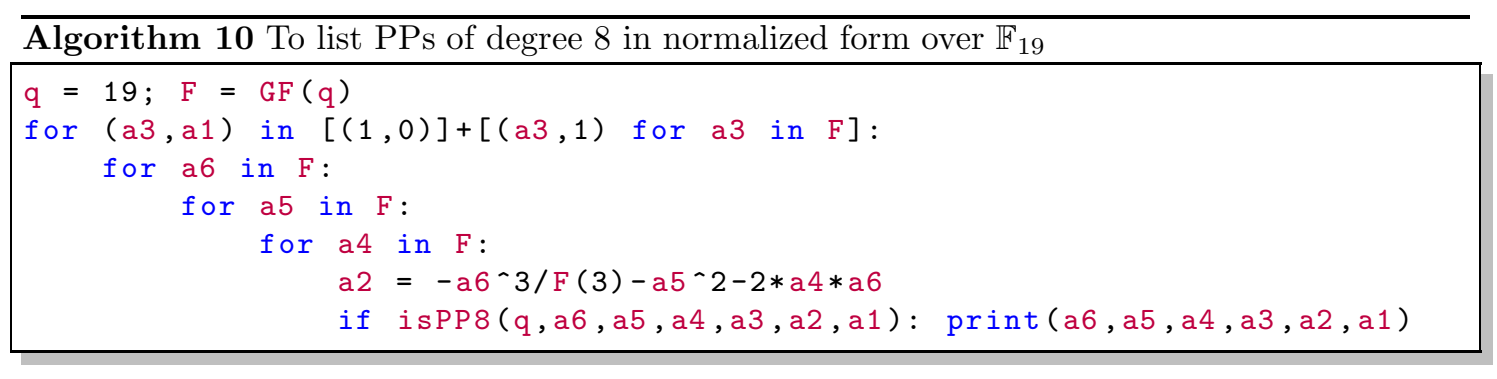

$\begin{array}{llll}(1,8,1,2,16,1), & (3,6,3,2,13,1), & (6,18,2,2,17,1), & (12,11,10,3,13,1), \\ (2,5,8,4,10,1), & (10,10,11,4,18,1), & (0,4,4,5,3,1), & (0,9,9,5,14,1), \\ (10,0,0,5,15,1), & (14,5,15,5,2,1), & (0,14,12,6,13,1), & (13,16,2,6,11,1), \\ (16,7,1,6,4,1), & (0,6,12,7,2,1), & (3,7,16,7,17,1), & (6,17,3,10,2,1), \\ (17,12,6,10,3,1), & (11,2,8,11,16,1), & (11,9,1,11,17,1), & (18,0,18,11,11,1), \\ (9,15,12,12,0,1), & (12,6,8,13,13,1), & (13,17,8,13,12,1), & (10,15,14,14,4,1), \\ (12,12,4,14,1,1), & (17,3,9,14,17,1), & (18,12,4,14,10,1), & (18,17,13,14,16,1), \\ (0,5,4,15,13,1), & (8,13,8,15,1,1), & (2,11,6,16,17,1), & (3,17,17,16,18,1), \\ (9,18,16,16,0,1), & (11,8,9,16,10,1), & (1,8,12,17,13,1), & (3,8,12,17,7,1), \\ (3,16,1,17,14,1), & (8,3,18,18,1,1), & (11,3,5,18,1,1), & (18,11,0,18,6,1) .\end{array}$

4.6. Case $q=13$. Note that $a_{4}=-a_{6}^{2} / 2$ since $\mathbf{H C}_{8}(13,2)=x_{6}^{2}+2 x_{4}$. We can make the following assumptions on $\left(a_{1}, a_{2}, \ldots, a_{6}\right) \in \mathbb{F}_{13}$, by linear transformations if necessary.

- Assume $a_{1} \in\{0,1\}$ as $q-1=12$ is coprime to 7 .

- When $a_{1}=0$, assume $a_{3} \in\{0,1\}$, as $q-1=12$ is coprime to 5 .

- When $a_{1}=a_{3}=0$, assume $a_{5} \in\{0,1,2,4\}$, as $\{1,2,4\}$ is a complete set of coset representatives of $\mathbb{F}_{13}^{*} /\left\{t^{3}: t \in \mathbb{F}_{13}^{*}\right\}$.

So Algorithm 11 lists PPs of degree 8 in normalized form over $\mathbb{F}_{13}$ up to linear transformations.

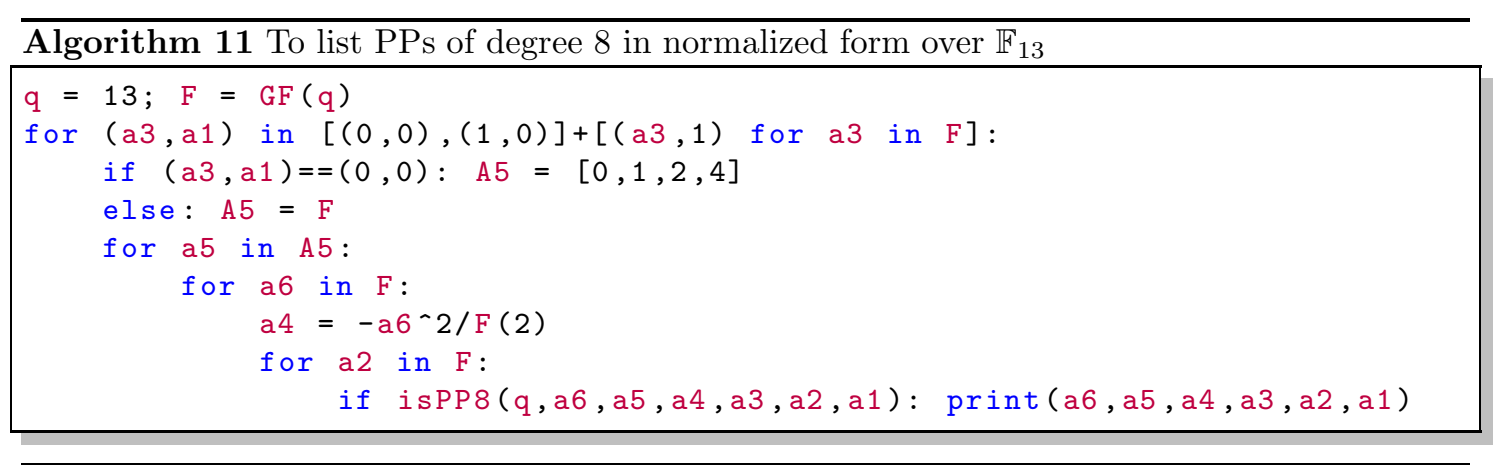

The output of Algorithm 11 prints 119 tuples $\left(a_{6}, a_{5}, \ldots, a_{1}\right)$, among which three tuples $(4,2,5,0,4,0),(10,2,2,0,4,0)$ and $(12,2,6,0,4,0)$ give three linearly related PPs of degree 8. Indeed, for $t=3 \in \mathbb{F}_{13}$, we have $t^{3}=1, t^{-1}=9$ and

$$
(10,2,2,0,4,0)=\left(4 t^{2}, 2 t^{3}, 5 t^{4}, 0,4 t^{6}, 0\right)=\left(12 t^{-2}, 2 t^{-3}, 6 t^{-4}, 0,4 t^{-6}, 0\right) .
$$


No other linear transformation relations exist among the outputting tuples. Therefore, there are exactly 117 linearly related classes of PPs of degree 8 over $\mathbb{F}_{13}$, as listed in Proposition 11 read off from the output of Algorithm 11.

Proposition 11. All PPs of degree 8 in normalized form over $\mathbb{F}_{13}$ are exactly those of the form $x^{8}+\sum_{i=1}^{6} t^{8-i} a_{i} x^{i}$, with $t \in \mathbb{F}_{13}^{*}$ and $\left(a_{6}, a_{5}, a_{4}, a_{3}, a_{2}, a_{1}\right)$ listed as follows:
$(0,1,0,0,5,0)$
$(0,1,0,0,7,0)$
$(4,2,5,0,4,0)$,
$(0,4,0,0,9,0)$,
$(2,0,11,1,12,0)$,
$(0,7,0,1,12,0)$,
$(7,7,8,1,9,0)$,
$(4,8,5,1,2,0)$,
$(3,1,2,0,2,1)$,
$(3,2,2,0,12,1)$,
$(10,5,2,0,1,1)$,
$(2,7,11,0,6,1), \quad(10,9,2,0,2,1)$,
$(4,11,5,0,8,1)$,
$(7,11,8,0,1,1)$,
$(12,11,6,0,1,1)$
$(4,2,5,1,10,1), \quad(4,3,5,1,7,1)$,
$(4,4,5,1,12,1)$,
$(7,5,8,1,5,1)$,
$(9,5,5,1,10,1), \quad(9,8,5,1,7,1), \quad(1,9,6,1,4,1)$,
$(12,10,6,1,3,1), \quad(8,0,7,2,11,1)$,
$(0,3,0,2,0,1)$,
$(7,4,8,2,12,1), \quad(1,8,6,2,5,1)$,
$(5,8,7,2,6,1)$,
$(1,9,6,2,3,1)$,
$(2,9,11,2,1,1)$,
$(6,12,8,2,4,1), \quad(8,1,7,3,12,1)$,
$(1,3,6,3,10,1)$,
$(1,4,6,3,12,1)$,
$(4,5,5,3,5,1)$,
$(8,5,7,3,7,1)$,
$(8,5,7,3,12,1)$,
$(12,5,6,3,8,1)$,
$(8,6,7,3,2,1)$,
$(9,12,5,3,7,1)$,
$(12,1,6,4,7,1), \quad(9,2,5,4,6,1)$,
$(4,4,5,4,5,1)$,
$(5,6,7,4,9,1)$,
$(1,10,6,4,6,1)$,
$(9,10,5,4,12,1),(10,10,2,4,9,1)$,
$(4,0,5,5,7,1)$
$(0,1,0,5,8,1)$,
$(3,5,2,5,12,1)$,
$(5,11,7,5,1,1), \quad(6,12,8,5,8,1)$,
$(10,12,2,5,4,1)$,
$(3,0,2,6,9,1)$,
$(10,0,2,6,11,1)$
$(5,2,7,6,10,1), \quad(6,7,8,6,3,1)$,
$(0,10,0,6,12,1), \quad(6,10,8,6,6,1)$,
$(11,11,11,6,2,1)$,
$(0,1,0,7,6,1), \quad(11,1,11,7,1,1)$
$(11,3,11,7,11,1),(4,4,5,7,6,1)$,
$(1,7,6,7,10,1)$,
$(10,7,2,7,10,1),(11$
$(11,10,11,7,2,1)$
$(0,0,0,8,1,1)$
$(12,0,6,8,2,1)$
$(1,1,6,8,1,1)$
$(9,8,5,8,1,1)$
$(1,11,6,7,4,1)$
$(9,10,5,8,10,1)$
$(7,11,8,8,1,1)$
$(8,11,7,8,10,1)$
$(7,1,8,9,2,1)$
$(4,9,5,8,2,1)$,
$(0,2,0,9,2,1)$,
$(7,2,8,9,5,1)$
$(12,6,6,9,8,1)$
$(3,9,2,9,2,1)$
$(11,1,11,9,0,1)$,
$(12,10,6,9,8,1)$,
$(1,11,6,9,5,1)$
$(4,12,5,9,0,1)$
$(7,12,8,9,0,1)$,
$(4,9,5,9,6,1)$,
$(12,1,6,10,4,1), \quad(0$,
$(0,3,0,10,3,1), \quad(9,4,5,10,5,1)$
$(4,5,5,10,5,1)$
$(5,1,7,10,10,1)$
$(10,5,2,10,9,1)$
$(10,7,2,10,0,1),(0,10,0,10,1,1)$
$(10,10,2,10,7,1),(5,12,7,10,9,1)$,
$(3,0,2,11,12,1)$
$(7,7,8,11,12,1)$ $(9,0,5,11,6,1), \quad(0,3,0,11,7,1), \quad(2,4,11,11,10,1),(2,6,11,11,0,1)$,
$(3,5,2,12,1,1)$
$(6,8,8,11,3,1)$
$(1,12,6,11,12,1),(8,3,7,12,2,1)$,
$(5,4,7,12,10,1)$
$(7,10,8,12,9,1)$
$(9,5,5,12,5,1), \quad(6,8,8,12,9,1)$,
$(4,9,5,12,0,1)$,
$(11,9,11,12,10,1)$,

4.7. Case $q=11$. Note that $a_{2}=-a_{5}^{2} / 2-a_{4} a_{6}$ since $\mathbf{H C}_{8}(11,2)=x_{5}^{2}+2 x_{4} x_{6}+2 x_{2}$. We can make the following assumptions on $\left(a_{1}, a_{2}, \ldots, a_{6}\right) \in \mathbb{F}_{11}$.

- Assume $a_{1} \in\{0,1\}$ as $q-1=10$ is coprime to 7 .

- When $a_{1}=0$, assume $a_{5} \in\{0,1\}$, as $q-1=10$ is coprime to 3 .

- When $a_{1}=a_{5}=0$, assume $a_{6} \in\{0,1,2\}$, as $\{1,2\}$ is a complete set of coset representatives of $\mathbb{F}_{11}^{*} /\left\{t^{2}: t \in \mathbb{F}_{11}^{*}\right\}$.

So Algorithm 12 lists PPs of degree 8 in normalized form over $\mathbb{F}_{11}$ up to linear transformations.

The output of Algorithm 12 prints 281 tuples $\left(a_{6}, a_{5}, \ldots, a_{1}\right)$. By Proposition 5 and our assumptions, linear transformation relations exist only among those with $a_{1}=a_{5}=0$, which are 


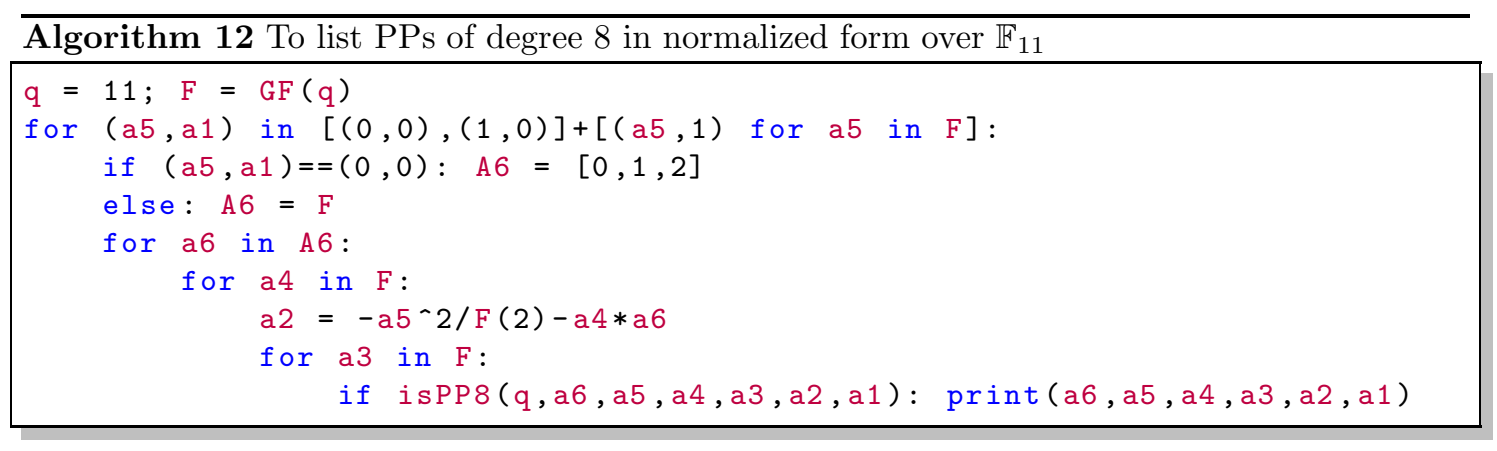

indeed the first eight tuples in the output, corresponding to four distinct linearly related classes. No other linear transformation relations exist among the outputting tuples. Therefore, there are exactly 277 linearly related classes of PPs of degree 8 over $\mathbb{F}_{11}$, as listed in Proposition 12 read off from the output of Algorithm 12.

Proposition 12. All PPs of degree 8 in normalized form over $\mathbb{F}_{11}$ are exactly those of the form $x^{8}+\sum_{i=1}^{6} t^{8-i} a_{i} x^{i}$, with $t \in \mathbb{F}_{11}^{*}$ and $\left(a_{6}, a_{5}, a_{4}, a_{3}, a_{2}, a_{1}\right)$ listed as follows:
$(0,0,0,2,0,0)$,
$(0,0,0,4,0,0)$,
$(2,0,6,2,10,0), \quad(2,0,7,3,8,0)$,
$(0,1,2,0,5,0)$,
$(1,1,1,0,4,0)$,
$(1,1,4,0,1,0)$,
$(1,1,4,5,1,0)$,
$(1,1,9,1,7,0)$,
$(1,1,10,5,6,0)$,
$(2,1,1,4,3,0)$,
$(2,1,4,0,8,0)$,
$(2,1,5,0,6,0)$,
$(3,1,2,3,10,0)$,
$(4,1,1,0,1,0)$,
$(4,1,6,4,3,0)$,
$(4,1,7,0,10,0)$,
$(4,1,8,3,6,0)$,
$(4,1,9,9,2,0)$,
$(4,1,10,9,9,0)$,
$(5,1,6,5,8,0)$,
$(6,1,6,5,2,0)$,
$(7,1,7,5,0,0)$,
$(8,1,0,0,5,0)$,
$(8,1,2,0,0,0)$,
$(9,1,3,4,0,0)$,
(9, 1, 5, 0, 4, 0),
$(9,1,5,5,4,0)$,
$(9,1,7,0,8,0)$,
(10, 1, 0, 9, 5, 0),
(0, 0, 1, 3, 0, 1),
$(0,0,2,9,0,1)$,
$(0,0,8,5,0,1)$,
$(2,0,3,5,5,1)$,
$(3,0,2,3,5,1)$,
$(3,0,4,1,10,1)$,
$(3,0,9,4,6,1)$,
$(3,0,10,1,3,1)$,
$(4,0,3,9,10,1)$,
(4, 0,8,4,1,1),
(5, 0, 10, 3, 5, 1),
$(6,0,2,4,10,1)$,
$(6,0,4,0,9,1)$,
(7, 0, 5, 0,9,1),
$(7,0,6,1,2,1)$,
$(8,0,5,9,4,1)$
$(9,0,3,1,6,1)$,
$(9,0,10,1,9,1), \quad(10,0,0,1,0,1)$,
$(10,0,1,4,1,1)$,
$(10,0,3,3,3,1)$
$(10,0,5,1,5,1), \quad(10,0,7,4,7,1), \quad(0,1,4,6,5,1)$,
$(1,1,9,10,7,1)$,
$(2,1,0,0,5,1)$,
$(2,1,10,10,7,1),(4,1,3,7,4,1)$,
$(4,1,5,5,7,1)$,
$(4,1,6,3,3,1)$,
$(5,1,2,5,6,1)$,
$(5,1,8,1,9,1)$
$(6,1,0,3,5,1)$,
$(6,1,0,6,5,1)$,
$(6,1,0,7,5,1)$,
$(8,1,1,3,8,1)$,
$(8,1,10,7,2,1), \quad(9,1,1,3,7,1)$,
$(9,1,2,3,9,1)$,
$(9,1,5,0,4,1)$,
$(9,1,6,7,6,1)$,
$(9,1,9,2,1,1)$,
$(10,1,0,7,5,1)$,
$(10,1,3,7,8,1)$,
$(10,1,7,2,1,1)$,
$(0,2,1,5,9,1)$
$(0,2,3,5,9,1)$,
$(1,2,4,2,5,1)$,
$(1,2,4,9,5,1)$,
$(1,2,7,6,2,1)$,
$(1,2,10,2,10,1)$,
$(2,2,5,5,10,1)$
$(4,2,0,7,9,1)$,
(4, 2, 5, 4, 0, 1),
$(5,2,2,9,10,1)$,
$(5,2,10,6,3,1)$,
$(6,2,0,4,9,1)$,
$(6,2,1,9,3,1)$,
$(6,2,8,4,5,1)$,
$(7,2,0,9,9,1)$,
$(7,2,1,5,2,1)$,
$(7,2,3,6,10,1)$,
$(8,2,2,5,4,1)$,
$(8,2,3,4,7,1)$,
$(8,2,8,4,0,1)$,
(9, 2, 1, 9, 0, 1),
$(9,2,6,6,10,1)$,
$(9,2,10,9,7,1)$,
$(10,2,10,5,8,1)$,
$(0,3,4,3,1,1)$,
$(0,3,5,8,1,1)$,
$(0,3,7,0,1,1)$,
$(0,3,8,0,1,1)$,
$(1,3,4,5,8,1)$,
(1, 3, 8, 0, 4, 1),
$(3,3,8,3,10,1)$,
$(3,3,8,8,10,1)$,
$(4,3,2,8,4,1)$,
$(4,3,8,5,2,1)$,
$(5,3,9,6,0,1)$,
$(6,3,4,4,10,1)$,
$(7,3,6,6,3,1)$,
$(7,3,8,3,0,1)$,
$(8,3,7,6,0,1)$,
(9, 3, 0, 4, 1, 1), 


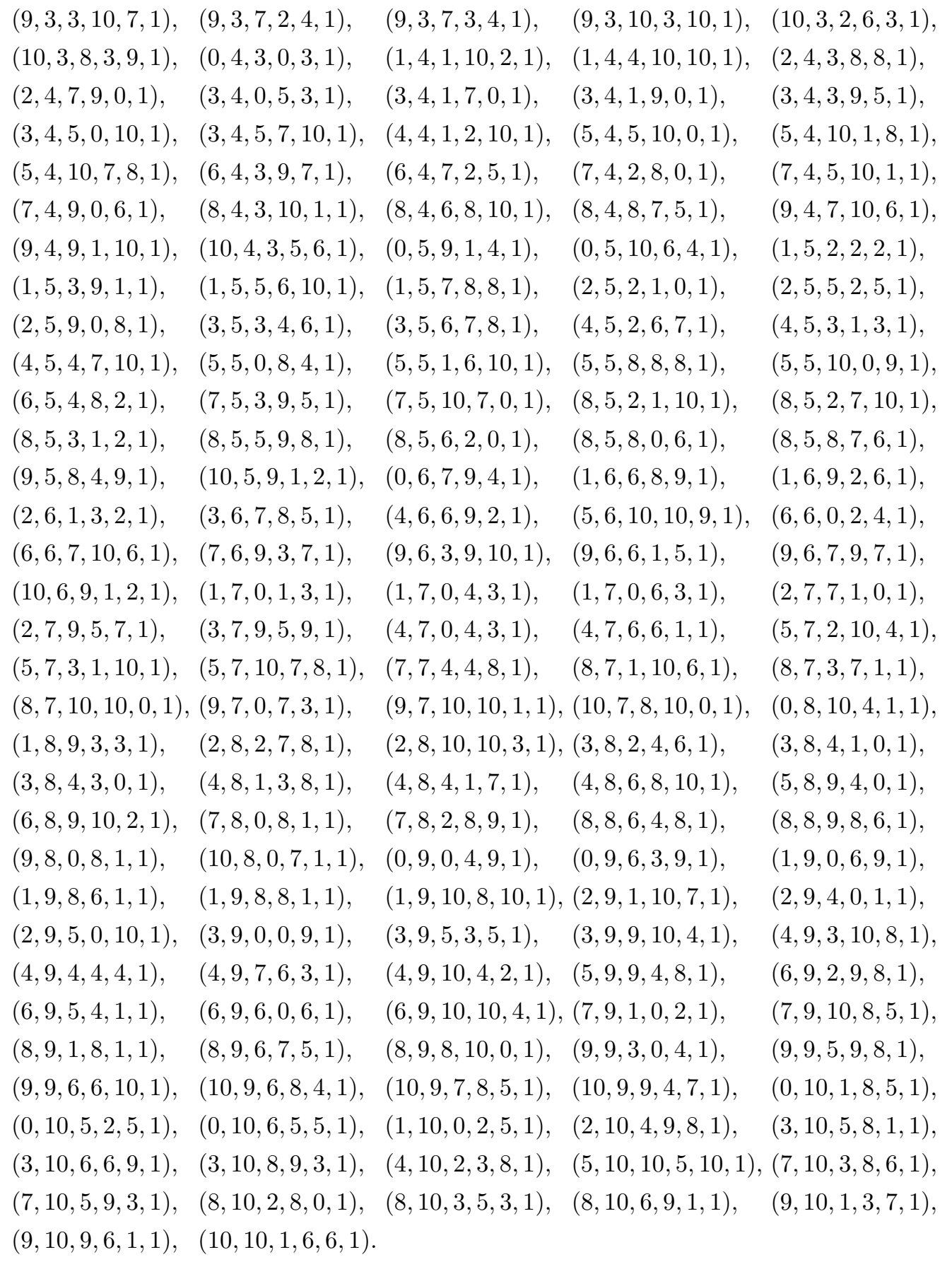

Acknowledgements. This work was partially supported by the Natural Science Foundation of Guangdong Province (No. 2018A030310080). The author was also sponsored by the National Natural Science Foundation of China (No. 11801579). Special thanks go to my lovely newborn daughter, without whose birth should this paper have come out much earlier. 


\section{REFERENCES}

[1] Chahal, J. S., \& Ghorpade, S. R. (2018). Carlitz-Wan conjecture for permutation polynomials and Weill bound for curves over finite fields. Finite Fields Appl., 54, 366-375. doi:10.1016/j.ffa.2018.07.006.

[2] Cohen, S. D., \& Fried, M. D. (1995). Lenstra's proof of the Carlitz-Wan conjecture on exceptional polynomials: an elementary version. Finite Fields Appl., 1, 372-375. doi:10.1006/ffta.1995.1027.

[3] Decker, W., Greuel, G.-M., Pfister, G., \& Schönemann, H. (2019). Singular 4-1-2 - A computer algebra system for polynomial computations. http://www.singular.uni-kl.de.

[4] Dickson, L. E. (1896/97). The analytic representation of substitutions on a power of a prime number of letters with a discussion of the linear group. Ann. of Math., 11, 65-120. doi:10.2307/1967217.

[5] Ding, C., \& Yuan, J. (2006). A family of skew Hadamard difference sets. J. Combin. Theory Ser. A, 113, 1526-1535. doi:10.1016/j.jcta.2005.10.006.

[6] Fan, X. (2018). A classification of permutation polynomials of degree 7 over finite fields. arXiv: 1812.02080.

[7] Fan, X. (2018). The Weil bound and non-exceptional permutation polynomials over finite fields. arXiv: 1811.12631.

[8] Fan, X. (2019). Permutation polynomials of degree 8 over finite fields of characteristic 2. arXiv: 1903.10309.

[9] Fried, M. D., Guralnick, R., \& Saxl, J. (1993). Schur covers and Carlitz's conjecture. Israel J. Math., 82, 157-225. doi:10.1007/BF02808112.

[10] von zur Gathen, J. (1991). Values of polynomials over finite fields. Bull. Austral. Math. Soc., 43, 141-146. doi:10.1017/S0004972700028860.

[11] Hayes, D. R. (1967). A geometric approach to permutation polynomials over a finite field. Duke Math. J., 34, 293-305. doi:10.1215/S0012-7094-67-03433-3.

[12] Hermite, C. (1863). Sur les fonctions de sept lettres. C. R. Acad. Sci. Paris, 57, 750-757.

[13] Hou, X.-d. (2015). Permutation polynomials over finite fields - a survey of recent advances. Finite Fields Appl., 32, 82-119. doi:10.1016/j.ffa.2014.10.001.

[14] Kemper, G. (2002). The calculation of radical ideals in positive characteristic. J. Symbolic Comput., 34, 229-238. doi:10.1006/jsco.2002.0560.

[15] Li, J., Chandler, D. B., \& Xiang, Q. (2010). Permutation polynomials of degree 6 or 7 over finite fields of characteristic 2. Finite Fields Appl., 16, 406-419. doi:10.1016/j.ffa.2010.07.001.

[16] Lidl, R., \& Niederreiter, H. (1997). Finite fields volume 20 of Encyclopedia of Mathematics and its Applications. (2nd ed.). Cambridge University Press, Cambridge. doi:10.1017/CB09780511525926 with a foreword by P. M. Cohn.

[17] Mullen, G. L. (Ed.) (2013). Handbook of finite fields. Discrete Mathematics and its Applications (Boca Raton). CRC Press, Boca Raton, FL. doi:10.1201/b15006.

[18] The Sage Developers (2017). SageMath, the Sage Mathematics Software System. URL: http://www .sagemath.org. doi:10.5281/zenodo. 593563.

[19] Wan, D. Q. (1993). A p-adic lifting lemma and its applications to permutation polynomials. In Finite fields, coding theory, and advances in communications and computing (Las Vegas, NV, 1991) (pp. 209-216). Dekker, New York volume 141 of Lecture Notes in Pure and Appl. Math.. URL: http://www.math.uci.edu/ dwan/lift.pdf.

E-mail address: fanx8@mail.sysu.edu.cn 